Ignazio Cassis, conseiller national et vice-président de la FMH, a été médecin cantonal au Tessin de 1996 à 2008. Avec le publiciste Ivo Giulietti, il a présenté en 2009 son livre, rédigé en italien: «ll Medico Cantonale. Dalla sua istituzione ai giorni nostri 1924-2008».

Les trois premiers chapitres de l'ouvrage décrivent l'évolution de la santé publique au Tessin de 1803 à 1924, ainsi que I'histoire des cinq médecins cantonaux qui se sont succédés à cette fonction depuis 1924. Ces chapitres sont une source inestimable de documents historiques captivants qui, à part les spécialistes, s'adressent cependant surtout à un public régional étant donné leur contenu spécifiquement cantonal.

En revanche, les chapitres 4 et 5 , de portée «supracantonale», ainsi que la préface du Prof. Thomas Zeltner, directeur de l'Office fédéral de la santé publique de 1991 à 2009, devraient intéresser un large public. Une traduction française* de ces chapitres est offerte à nos lecteurs dans le présent et le prochain numéro du BMS.

La première partie ci-après comprend la préface ainsi que le chapitre 4 . L'essentiel de ce chapitre traite de la mission et du travail quotidien de tout médecin cantonal, indépendamment du canton qui l'emploie: qu'est-ce qu'un médecin cantonal, en quoi consistent ses tâches quotidiennes, à quels défis est-il confronté, comment cette fonction est-elle appelée à évoluer?

La rédaction

* La traduction française de l'introduction et des chapitres 4 et 5 de l'ouvrage a été rendue possible par un financement de Sanofi et de Merck Sharp \& Dohme-Chibret. Les auteurs et la rédaction remercient ces deux entreprises de leur généreux soutien.

\section{Le livre «Le médecin cantonal» d'Ivo Giulietti et Ignazio Cassis}

\section{Préface de Thomas Zeltner}

L'éventail des maladies humaines, et avec lui nos connaissances sur la santé, ont fortement changé depuis 120 ans. À l'époque, l'espérance de vie moyenne en Suisse était de 41 ans, et les infections figuraient en tête des causes de maladie et de décès.

En ce temps-là (vers 1848) où la Suisse se constituait en un État moderne, le monde était frappé par des épidémies majeures: c'est ainsi que six grandes vagues de choléra ont frappé l'Europe au $19^{\text {e }}$ siècle. Celle qui a débuté en 1884 a tué en trois ans plus de 200000 personnes en France, en Italie et en Espagne. En 1886, 182 personnes en Suisse sont mortes de la variole; 9 ans auparavant, une épidémie de typhus pétéchial avait ravagé les Balkans. Pourtant, protéger la santé de la population n'était pas une priorité dans les années où naissait notre État fédéral. La Constitution fédérale suisse n'accordait à la Confédération que des compétences limitées dans le domaine de la santé: le système suisse de santé était, et reste aujourd'hui encore, marqué par une forte autonomie cantonale.

Les épidémies de variole et de choléra n'ont pas épargné le Tessin. Elles étaient généralement introduites de Lombardie voisine ou d'Italie du Nord. La dernière vague de choléra au Tessin, en 1867, a frappé 221 personnes dont 122 sont décédées. Cette situation a abouti en 1870 à la promulgation d'une loi qui rendait obligatoire la création de dispensaires d'arrondissement. Ceux-ci devaient assurer une prise en charge médicale gratuite, à l'exception des interventions chirurgicales majeures. Le législateur voulait garantir des traitements globalement efficaces à un coût abordable, qui agissent au moment crucial et profitent aussi aux personnes vivant dans des lieux reculés. Cela correspondait au fond au système obligatoire de soins de santé accessibles à tous, avec catalogue complet de prestations, mis en place une centaine d'années plus tard.

Malgré la forte empreinte cantonale sur le système suisse de santé, la nécessité d'une réglementation et d'une coordination au niveau fédéral est apparue assez rapidement, notamment dans les domaines dépendant du trafic intercantonal et international ou fortement influencés par les progrès scientifiques et techniques. Ainsi, l'instauration en 1877 de la libre circulation des médecins en Suisse et l'introduction d'un diplôme fédéral pour les médecins, les vétérinaires et les pharmaciens, ont clairement consacré la volonté de réglementer en priorité le volet curatif de l'activité médicale. Néanmoins, la création d'un office fédéral de la santé n'a pas tardé à s'imposer quand plusieurs conférences internationales ont consacré la nécessité impérative de lutter contre les épidémies de variole et qu'un premier projet de loi sur les épidémies en 1882 a été rejeté en votation populaire. L'introduction de la loi sur les épidémies en 1886 et la persistance de fortes épidémies de variole ont finalement conduit le Conseil fédéral à décider, le 28 juin 1893, de créer l'Office fédéral de la santé publique. Celui-ci était composé à l'origine du directeur, d'un adjoint et d'un chancelier.

C'est donc à l'époque de la création de l'OFSP que remonte son mandat de lutter contre les épidémies, auquel s'est ajouté en 1905 le contrôle des denrées avec l'introduction de la loi sur les denrées alimentaires. La loi fédérale du 18 décembre 1970 sur la lutte contre les maladies transmissibles de l'homme (loi sur les épidémies, LEp) a été promulguée à la suite d'une grave épidémie de typhus qui a frappé Zermatt en 1963 (plus de 400 cas et plusieurs morts, parmi lesquels de nombreux touristes étrangers). Cette loi a renouvelé le cadre juridique de la lutte contre les 


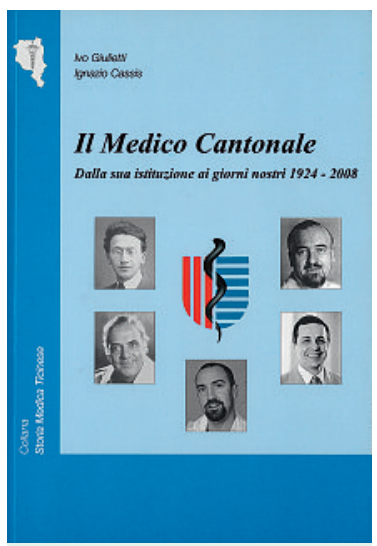

Ivo Giulietti, Ignazio Cassis II Medico Cantonale Dalla sua istituzione ai giorni nostri 1924-2008.

Taverne: Storia Medica Ticinese; 2009

240 pages. $48 \mathrm{CHF}$. ISBN 978-88-902131-5-9 maladies transmissibles et a institué, avec la création du Service du médecin cantonal, une interface nécessaire entre la Confédération et les cantons et un lien important entre santé publique et santé individuelle.

Aujourd'hui, les activités tendent à se focaliser sur l'observation épidémiologique des maladies transmissibles. En effet, la mobilité croissante de la population depuis le milieu du $20^{\text {e }}$ siècle (hausse du nombre de voyageurs et de la rapidité des transports) a également augmenté le risque de propagation des maladies infectieuses. La densification globale des réseaux et des personnes a donc un impact croissant sur la politique de santé, en Suisse comme ailleurs. Le trafic intense des voyageurs et des marchandises avec l'étranger nous contraint à harmoniser en permanence notre législation avec celle de nos voisins, notamment de l'Europe communautaire. L'évolution qu'a connue la Suisse au niveau cantonal et national au cours du $19^{\mathrm{e}}$ et du $20^{\mathrm{e}}$ siècle, à savoir la prise de responsabilité par l'État et la mise en place d'institutions nationales, s'est répétée en 2005 dans l'Union Européenne avec la création du Centre européen de prévention et de contrôle des maladies (ECDC) en conséquence directe de la crise du SRAS. La situation géographique et économique de la Suisse nous impose aussi de resserrer et d'intensifier notre collaboration avec les institutions de l'UE dans le domaine de la santé. La libre circulation des personnes dans l'espace européen, la suppression des frontières intra-européennes et les échanges intensifs de produits et de denrées alimentaires recèlent toujours de nouveaux dangers potentiellement plus graves pour la santé.

Dans le même temps, et c'est légitime, la population devient toujours plus exigeante en ce qui concerne l'amélioration de la sécurité et la diminution des risques pour la santé. Si on laisse de côté l'explosion des coûts des soins curatifs, c'est dans ces domaines que se posent les défis majeurs à la santé publique, qui ne pourront être résolus que dans le cadre d'une action conjointe internationale. Pour autant, les cantons et par conséquent les médecins cantonaux ne pourront se dérober à leur rôle de gardiens périphériques de la santé publique en Suisse, et c'est à eux qu'il appartiendra de relever ces défis et d'en faire un atout. De ce point de vue, une rétrospective historique telle qu'elle est présentée dans cet ouvrage offre toujours une excellente occasion d'entamer une réflexion dans un effort de tirer les enseignements du passé pour s'ouvrir aux anciens et nouveaux défis qui se posent dans le domaine de la santé. 